2. Palella FJ Jr, Delaney KM, Moorman AC, Loveless MO, Fuhrer J, Satten GA, et al. Declining morbidity and mortality among patients with advanced immunodeficiency virus infection. N Engl J Med 1998; 338:853-60.

3. Burman WJ, Reves RR, Cohn DL. The case for conservative management of early HIV disease. JAMA 1998; 280:93-5.

4. UNAIDS. The Joint United Nations Programme on HIV/AIDS. Report on the global HIV/AIDS epidemic: June 1998. (www.us.unaids.org)

5. Makadon HJ, Silin JG. Prevention of HIV infection in primary care: current practices, future possibilities. Ann Intern Med 1995;123:715-9.

6. Hecht FM, Grant RM, Petropoulos CJ, Dillon B, Chesney MA, Tian H, et al. Sexual transmission of an $H I V-1$ variant resistant to multiple reverse-transcriptase and protease inhibitors. $\mathrm{N}$ Engl J Med 1998;339:307-11.

7. Cohen OJ, Fauci AS. Transmission of multidrug-resistant human immunodeficiency virus, the wake-up call. N Engl J Med 1998;339:341-3.

8. HIV prevention through early detection and treatment of other sexually transmitted diseases-United States. Recommendations of the Advisory Committee for HIV and STD prevention. MMWR Morb Mortal Weekly Rep 1998;47(RR-12):1-24.

9. Case-control study of HIV seroconversion in healthcare workers after percutaneous exposure to HIV-infected blood-France, United Kingdom, and United States, January 1988-August 1994. MMWR Morb Mortal Weekly Rep 1995;44:929-33.

10. Katz MH, Gerberding JL. Postexposure treatment of people exposed to the human immunodeficiency virus through sexual contact or injection-drug use. $N$ Engl J Med 1997;336:1097-100.

11. Pinkerton SD, Holtgrave DR, Pinkerton HJ. Costeffectiveness of chemoprophylaxis after occupational exposure to HIV. Arch Intern Med 1997;157: 1972-80.

\section{The Tyranny of Names in Mental Health Care}

Yogi Berra's wisdom often extends beyond baseball. He once said that 90 percent of baseball is half mental, but he could have as easily said that 90 percent of family practice is half mental illness. Family physicians spend a considerable portion of their time and effort in the diagnosis and treatment of emotional distress and clinical psychiatric

Submitted 10 November 1998.

From the Department of Family Medicine, University of Michigan Health System, Ann Arbor, Michigan. Address reprint requests to Thomas $L$. Schwenk, MD, Department of Family Medicine, 1018 Fuller St, Ann Arbor, MI 48109. disease. ${ }^{1}$ Most prescriptions for psychotropic medications are written by family physicians, ${ }^{2}$ and most patients who receive mental illness care do so from primary care physicians rather than specialty mental health services, leading to the labeling of primary care as the "hidden mental health care system." 3

The occurrence of both distress and mood and anxiety disorders in primary care is high, their functional impact profound, their presence frequently undiagnosed by primary care and family physicians, and their diagnosis often deliberately miscoded because of insurance restrictions and social stigma. ${ }^{4}$ These problems and deficiencies have led to a rigorous, but increasingly unproductive, line of linear reasoning that suggests the major solution to this epidemic of undiagnosed and presumably untreated mental illness is to exhort physicians to perform better through education about diagnostic criteria and appropriate treatment, usually in the form of guidelines and protocols. ${ }^{5}$

Recent studies, however, have suggested the futility of these educational exhortations, as well as the reasons for this futility. Many cases of depression, for example, might meet diagnostic criteria but are mild and cause little functional impact. ${ }^{6}$ Family physicians have competing priorities for the care of most patients and must decide which of several problems deserve attention during a short visit. ${ }^{7}$ Depression often is watched or otherwise not treated in a specific fashion because of patient resistance and the presence of seemingly more serious problems. Treatment provided to many depressed primary care patients does not lead to improvement, and patients whose depression is not diagnosed often do as well as or better than those whose depression is diagnosed, because of the waxing and waning course of depression in primary care. ${ }^{8}$ Prognosis has more to do with whether the patient has a chronic medical disease and the patient's marital and social support than the specific nature of the depression treatment. ${ }^{9}$ In sum, depression as it is known, described, labeled, and depicted in psychiatry might have little in common with the disease that carries the same name in primary care.

We are prisoners of how medical problems are named. The clinical skills and outcomes of primary care physicians have been unfairly compared with those of psychiatrists and mental health workers 
because of the belief that psychiatrists and primary care physicians are treating the same problem. After all, it has the same name. What we are actually describing with diagnostic names are syndromes, groups of symptoms that seem to hang together. Most of what we diagnose in psychiatry are syndromes, which are valid only to the extent they are drawn from the same epidemiologic world. The problem is that criterion-based syndromes were drawn from the psychiatric world and later applied to primary care. The tyranny of the name lingers because of the circular reasoning that begins with a need for a name of the disease in question.

A problem must have a name before it can be studied. It must be studied before diagnostic criteria of adequate sensitivity and specificity can be established. Diagnostic criteria are essential before the benefits and harms of treatment protocols can be developed. Treatment protocols are necessary to learn which standardized approaches are more likely to improve the patient's quality and quantity of life. A likelihood of improvement is necessary to decide whether the disease deserves treatment. And we must know if it is worth treating before we know whether it is worthy of a name.

The study by Nease and colleagues ${ }^{10}$ in this issue of $\mathcal{F} A B F P$ can provide some help in freeing family physicians from the Escher-like shackles of nomenclature in mental illness. What Nease and his colleagues have done is to throw away the names and start again by asking a simple question: How do mental illnesses cluster in their functional impact on patients, irrespective of their names or diagnostic criteria? Through cross-sectional cluster analyses of standardized questionnaire data regarding mood and anxiety symptoms, DSM-III-R diagnoses, and health-related quality of life, they found that patients could be organized into four groups that differed from each other on sociodemographic indicators, health-related quality of life, mental and physical functioning, and prevalence of criterion-based psychiatric disorders. This clustering fits well with the experience of family physicians who have been found to care for patients, not on the basis of criterion-based diagnostic names, but on the basis of severity and functional impact.

Nease and colleagues have organized that intuitive approach into four discrete categories spread across a spectrum of functional impact. On one end is a high-functioning group with a high likelihood of being employed, a low likelihood of being disabled, a higher level of education, a higher household income, and a lower rate of chronic health problems. On the other end is a low-functioning group characterized by a high proportion of women with a high level of unemployment, disability, poverty, and chronic ill health. Two groups in the middle have intermediate levels of work disability, household income, chronic health problems, and mental and physical health functioning, both with moderate levels of anxiety but one featuring more severe mood disturbance. Several additional interesting features emerge from this scaled functional nomenclature; for example, the most severely affected group has a markedly higher level of cigarette smoking than the others. From high functioning to low functioning, the proportion of women in the groups increased significantly. The likelihood of almost every depressive or anxiety disorder increased from cluster 1 to cluster 4 , as did a range of vague somatic symptoms that characterize the practice of primary care.

The potential implications of this groundbreaking, albeit preliminary, study are large. This cluster analysis confirms the high level of comorbidity between depressive and anxiety symptoms, the artificial separation of which has confused physicians for generations. Instead of discrete diagnostic labels, such as major depressive disorder or generalized anxiety disorder, we might label a patient as having, for example, "emotional distress NOS of minimal functional impact," or "affective disorder NOS of significant functional impact." Family physicians would know the first category as the worried well, to which is applied a watchfulwaiting approach with assessment of new symptoms and functional impact that might require future treatment. The two middle groups might correspond to the false-positive and false-negative patients described by Klinkman et al, ${ }^{11}$ patients who might soon become sufficiently depressed to require treatment or whose depression might be remitting, as is the natural history of the disease in primary care. The low-functioning category is currently labeled as severely depressed, for which family physicians are fairly accurate in their detection and treatment.

This functional nomenclature also explains the known phenomenon of prescribing antidepressants in dosages sufficient to improve function, 
whether therapeutic or not, another source of purported poor performance by primary care physicians that might be shown to be justified by future empiric research. These clusters also suggest that the frequent use of a standardized assessment of function, such as the SF-36 Health Survey, would be more useful than depression questionnaires appear to be. Chronic medical illness would prompt a more thorough search for functionally important mental illness, which would be more likely to be treated under this nomenclature than it is when we fuss about whether diagnostic symptoms are due to depression or to its concomitant medical disease.

Nease and colleagues have shown that how mental illness is named not only might be inaccurate and misleading but could detract from the likelihood that important psychiatric problems receive appropriate attention. Psychiatric syndromes have been exported into primary care, and they do not fit, but the names have taken on a life of their own and have outlived their usefulness in primary care practice. They do not reflect the range of severity of mental illness seen in primary care and confuse rather than enlighten the process of assessing the results of treatment. In Romeo and fuliet, Shakespeare wrote, "What's in a name? A rose by any other name would smell as sweet," but Nease and colleagues have shown that how depression and mental illness are named can profoundly influence their understanding and importance and ultimately the accuracy of their diagnosis and their likelihood of successful treatment.

Thomas L. Schwenk, MD Ann Arbor, Mich

\section{References}

1. Katon W, Schulberg H. Epidemiology of depres- sion in primary care. Gen Hosp Psychiatry 1992; 14:237-47.

2. Pincus HA, Tanielian TL, Marcus SC, Olfson M, Zarin DA, Thompson J, et al. Prescribing trends in psychotropic medications: primary care, psychiatry, and other medical speciaties. JAMA 1998;279:526-31.

3. Regier DA, Narrow WE, Rae DS, Manderscheid RW, Locke BZ, Goodwin FK. The de facto US mental and addictive disorders service system. Epidemiologic catchment area prospective 1-year prevalence rates of disorders and services. Arch Gen Psychiatry 1993;50:85-94.

4. Rost K, Smith R, Matthews DB, Guise B. The deliberate misdiagnosis of major depression in primary care. Arch Fam Med 1994;3:333-7.

5. Depression in primary care. Vol 2, treatment of major depression. Clinical practice guideline, no 5. Rockville, Md: US Department of Health and Human Services, Public Health Service, Agency for Health Care Policy and Research; 1993. AHCPR publication 930551.

6. Coyne JC, Fechner-Bates S, Schwenk TL. Prevalence, nature, and comorbidity of depressive disorders in primary care. Gen Hosp Psychiatry 1994;16: 267-76.

7. Schwenk TL. Competing priorities and comorbidities. So much to do and so little time. Arch Fam Med 1997;6:238-9.

8. Simon GE, Lin EH, Katon W, Saunders K, VonKorff M, Walker E, et al. Outcomes of "inadequate" antidepressant treatment. J Gen Intern Med 1995; 10:663-70.

9. Coyne JC, Klinkman MS, Gallo SM, Schwenk TL. Short-term outcomes of detected and undetected depressed primary care patients and depressed psychiatric patients. Gen Hosp Psychiatry 1997;19:333-43.

10. Nease DE, Volk RJ, Cass AR. Investigation of a severity-based classification of mood and anxiety symptoms in primary care patients. J Am Board Fam Pract 1999;12:21-31.

11. Klinkman MS, Coyne JC, Gallo S, Schwenk TL. False positives, false negatives, and the validity of the diagnosis of major depression in primary care. Arch Fam Med 1998;7:451-61. 